Relations industrielles

Industrial Relations

\title{
Les employés de bureau et les négociations collectives
}

\section{Léo Roback}

Volume 14, numéro 1, janvier 1959

URI : https://id.erudit.org/iderudit/1022339ar

DOI : https://doi.org/10.7202/1022339ar

Aller au sommaire du numéro

\section{Éditeur(s)}

Département des relations industrielles de l’Université Laval

\section{ISSN}

0034-379X (imprimé)

1703-8138 (numérique)

Découvrir la revue

\section{Citer ce document}

Roback, L. (1959). Les employés de bureau et les négociations collectives. Relations industrielles / Industrial Relations, 14(1), 68-75.

https://doi.org/10.7202/1022339ar

Tous droits réservés @ Département des relations industrielles de l’Université Laval, 1959
Ce document est protégé par la loi sur le droit d'auteur. L'utilisation des services d'Érudit (y compris la reproduction) est assujettie à sa politique d'utilisation que vous pouvez consulter en ligne.

https://apropos.erudit.org/fr/usagers/politique-dutilisation/ 


\section{Les employés de bureau et les négociations collectives}

\section{LÉo RoBACK}

Les employés de bureau s'accroissent proportionnellement plus vite que les travailleurs manuels mais ils comptent le plus faible pourcentage de syndiqués. Les avantages relatifs qui étaient le lot traditionnel des collets-blancs se raréfient. Le travail des collets-blancs se rapproche sans cesse de celui qu'effectue l'employé d'usine. Le syndicalisme rencontre des obstacles parmi ces employés, mais il demeure la seule formule capable de redonner aux collets-blancs leur véritable dignité.

La Section des relations industrielles du Montreal Board of Trade a organisé une discussion au sujet de la négociation collective et les employés de bureau. C'est là un sujet qui intéresse hautement le patronat comme le mouvement syndical et le public en général. Cette question attire une attention croissante aux Etats-Unis depuis quelques temps et, j'espère, fera le sujet d'une discussion plus large au Canada également.

\section{LE CONTEXTE DU PROBLÈME}

Deux facteurs principaux ont servi à mettre la question du syndicalisme chez les employés de bureau à l'ordre du jours des affaires publiques. D'abord, cette catégorie d'employés constitue aujourd'hui une proportion de la main d'oeuvre bien plus considérable qu'auparavant, et la tendance continue vers la hausse. Deuxièmement, les employés de bureau constituent actuellement le plus fort groupe à n'être que partiellement syndiqué. On estime qu'il y a environ 650,000 employés de bureau au Canada à l'heure actuelle. Près de la moitié d'entre eux sont employés par les entreprises dans l'industrie manufacturière. Les gouvernements et les institutions financières (banques, compagnies d'assurances, etc.) comptent également des groupes considérables de ces employés. La question du syndicalisme et des négociations collectives chez les employés gouvernementaux est compliquée par des facteurs particuliers, et c'est pourquoi je m'en tiendrai ici aux seuls employés de bureau en dehors du service gouvernemental.

Dans quelle mesure les employés de bureau au Canada sont-ils syndiqués? Malheureusement, on ne dispose d'aucun renseignement complet, précis et à date à ce sujet. Un relevé du Ministère canadien du travail, en 1951, traitant exclusivement des industries manufacturières, indiquait que quelques 14,000 employés de bureau étaient alors assujettis au régime d'une convention collective.

L'on estime dans certains milieux syndicaux (union internationale) 
que quelque 40,000 employés de bureau sont syndiqués à l'heure actuelle, dans toutes les industries. On peut dire que parmi les travailleurs manuels syndicables, environ 40 pour cent d'entre eux sont syndiqués, tandis que seulement 10 pour cent des employés de bureau le sont, dont un grand nombre se retrouvent dans des organisations indépendantes en dehors des principaux mouvements ouvriers.

Il est évident que cet état de choses constitue à la fois un défi et un problème pour le mouvement syndical. En présence de cet accroissement proportionnel rapide du nombre d'employés de bureau par rapport aux travailleurs industriels, une question urgente se pose au mouvement syndical. PEUT-ON ORGANISER LES EMPLOYES DE BUREAU? COMMENT ORGANISER LES EMPLOYES DE BUREAU?

\section{LES EMPLOYÉS DE BUREAU ONT-ILS BESOIN D'UN SYNDICAT}

Plusieurs répondront peut-être: Non! Il convient de signaler cependant que les milieux patronaux discutent beaucoup de cette question depuis quelques mois. Un éditorial paru il y a un an dans le New York Times déclara:

\section{\& Les collets-blancs gagnent du terrain rapidement, du point de vue nombre, mais sont devancés, du point de vue salaires, par rapport aux autres groupes dans la main-d'oeuvre américaine. 》 (30 décem- bre 1956)}

M. C. C. Belden, un expert patronal en matière de relations industrielles, a conseillé aux employeurs qui voudraient garder leurs employés de bureau à l'écart des unions ouvrières (c'est-à-dire la majorité des employeurs) de donner aux collets-blancs des avantages AU MOINS EGAUX A CEUX DONT JOUISSENT LES TRAVAILLEURS D'USINES. Adressant la parole à une réunion de gérants de bureau à Hamilton, M. Belden a signalé que le travail de bureau, autrefois le plus désirable, offre maintenant des conditions moins désirables que le travail de production. Et M. Belden de continuer:
«L'employé de bureau a souvent été oublié en ce qui concerne le salaire, le temps supplémentaire les primes d'équipe, les congés et vacanoes payés, les augmentations de mérite et les avantages margi- naux. Si vous voulez éviter l'organisation syndicale, vous dervrez agir en sorte que vos employés de bureau préféreront vous à l'union. » (Hamilton Spectator, 9 janvier 1957.)

Il n'y a aucun doute que les travailleurs manuels rattrappent les collets-blancs dans le domaine des conditions de travail, et les devancent dans celui des salaires. Cette comparaison prend un grand relief, surtout, lorsqu'on compare les avantages obtenus par les travailleurs SYNDIQUES à ceux que reçoivent les employés de bureau NONSYNDIQUES. Citons à ce propos M. Howard Coughlin, présidentgénéral de l'Union internationale des employés de bureau:

«Dans beaucoup de cas, les travailleurs manuels syndiqués ont obtenu des avantages marginaux qui dépassent ceux qu'ont reçu les 
collets-blancs non-organisés. 》 (Journal of Commerce, 4 novembre 1957.)

Regardons, comme exemple de cette tendance, la question des heures de travail. A la fin de la dernière guerre, la semaine normale de travail dans les usines était généralement de 48 heures ou plus, et la semaine de 6 jours était la règle générale. Les employés de bureaı dans les industries manufacturières avaient déjà la semaine de 5 jours, pour la plupart, et la semaine de $371 / 2$ à 40 heures était assez généralisée. Or, depuis 1945 , et surtout depuis 5 ou 6 ans, les travailleurs. syndiqués ont réussi à établir la semaine de 5 jours, 40 heures de travail comme la norme. Ainsi, le Relevé du Ministère du travail de 1957 sur les conditions de travail démontre que 88 pour cent des travailleurs d'usine ont la semaine de 5 jours, en comparaison de 92 pour cent chez les employés de bureau. La différence n'est guère impressionnante! Toujours au chapitre des heures de travail, on constate que les travailleurs manuels jouissent d'un grand avantage par rapport au pauvre collet-blanc non-syndiqué, et c'est au sujet de la prime pour travail supplémentaire. Presque tous les travailleurs d'usine sont payés au taux de temps et demi pour le surtemps, mais tel n'est pas le cas pour la majorité des employés de bureau bien que l'on constate une certaine amélioration récente à cet égard. Ainsi, par exemple, les banques, inspirées et encouragées sans doute par une campagne d'organisation syndicale parmi leurs employés, ont, vers la fin de 1956, inauguré le paiement du salaire au taux de temps et demi après $40^{\circ}$ heures de travail.

Les collets-blancs sont encore en avant de leurs confrères en salopettes, les premiers recevant 2 semaines de vacances après un an de service, 8 congés statutaires payés et bénéficient du congé-maladie. Mais l'écart sur ces items décroît de plus en plus entre les deux groupes de travailleurs.

\section{Et LES SALAIRES?}

Il est difficile d'établir des barèmes précis pour comparer les taux de salaires des travailleurs d'usine et des collets-blancs. Il est intéressant toutefois de comparer les taux fixés pour certaines occupations de bureau typiques avec les taux établis pour les journaliers d'usine. Selon le relevé annuel de 1957 du Montreal Board of Trade, le traitement moyen à Montréal, en octobre 1957, pour commis de bureau intermédiaire était de $\$ 63.74$ par semaine. Pour les commis préposés à la facturation, la moyenne était de $\$ 61.20$ par semaine. Il s'agit des traitements moyens pour employés masculins détenant des positions numériquement importantes, et exigeant une expérience et une responsabilité considérable. OR on trouve nombre d'usines organisées à Montréal où un simple journalier touche AUTANT OU MEME PLUS que le traitement moyen pour commis intermédiaires ou préposés à la facturation. En voici quelques exemples basés sur le salaire hebdo- 
madairement payé aux journaliers: Impérial Oil $-\$ 70.80$; Canada Cement - \$68.00; Dow Breweries - \$75.60; Stelco - \$65.40; SherwinWilliams - $\$ 64.00$; Canadian Car - $\$ 60.00$; Dominion Bridge $\$ 61.60$ American Can - $\$ 76.00$; Crane Ltd. - $\$ 64.60$; Montreal Locomotive - \$68.40; Imperial Tobacco - \$67.60; Canadian Tube - \$63. 40; Canada Packers - \$63.20; RCA Victor - $\$ 60.80$; Hinde \& Dauch - $\$ 60.00$; Canadian Schenley (Valleyfield) $-\$ 67.20$, et ainsi de suite.

Il me semble qu'il y a quelque chose qui cloche lorsqu'un homme responsable pour les factures ne touche que le même salaire que reçoit un balayeur dans une usine, et parfois moins. Et que dire du cas d'un caissier à la banque, responsable de quelque $\$ 60,000$ par jour, qui touche le salaire princier de $\$ 39$. par semaine! (Globe and Mail, 6 décembre 1956)

LE COLlet-BLANC ET Le TRAvaIlleur

L'un des aspects les plus frappants de l'évolution industrielle actuelle est la diminution croissante de la différence entre le travail de l'employé de bureau et celui du travailleur d'usine. Un nombre croissant d'employés de bureau sont des OPERATEURS DE MACHINES (IBM, Key punch, machines de comptabilité, etc.), tandis que l'automatisation transforme des travailleurs en collets-blancs, dont le travail consiste principalement en la surveillance des appareils électroniques. Plus frappant encore est la mise en vigueur, DANS LES BUREAUX, de systèmes et techniques jusqu'ici utilisés seulement dans les usines. Ainsi, on trouve le chronométrage et le génie industriel appliqués au travail de l'employé de bureau. Celui-ci voit son travail analysé et fractionné en «opérations » et en «éléments 》, mesurés en minutes et en secondes. Le dernier numéro de "Canadian Business 》, organe de la Chambre de Commerce du Canada, contient un exposé fort élaboré des méthodes utilisées pour "augmenter l'efficacité des employés de bureau». On pourrait bien consacrer tout un livre aux implications sociologiques et psychologiques de ces développements. On se demande, par exemple, ce que ça peut faire à un employé de bureau expérimenté de voir son travail fractionné en petits morceaux comme suit:

«Compter des items sur la feuille - temps par unité - 0065 heure, 9,240 item par heure», ou bien «préparer les chèques de la. liste de paie sur une machine Burroughs, 100 opérations par heure ». P.M. Grieve, un ingénieur qui expose les détails du «Clerical Work Standards Method》 dans le «Management Review》 de mars 1956, avoue que la nouvelle méthode, tout en faisant des économies pour l'employeur, pourrait affecter le moral de l'employé et pourrait même affaiblir sa loyauté envers l'employeur et encourager l'organisation syndicale. Je crois que M. Grieve a raison. 
POURQuOI LES DIFFicultÉS À ORGANISER LES EMPLOYÉS DE BUREAu?

Il ne suffit pas de répondre que les employeurs ne sont pas généralement en faveur de l'organisation de leurs employés de bureau. Après tout, presque toutes les grandes campagnes d'organisation dans l'industrie ont réussi sans beaucoup d'enthousiasme ou d'encouragement de la part des patrons. Mais pour comprendre les raisons pour lesquelles le syndicalisme progresse plutôt lentement chez les colletsblancs, il faut examiner quelques uns des facteurs, objectifs et subjectifs, qui affectent l'attitude et la mentalité des collets-blancs. Inutile d'ajouter qu'il faut procéder avec prudence lorsqu'on essaie de faire des généralisations sur la conduite humaine. Evidemment, la situation diffère de place en place, selon la situation concrète. Cependant, pour fins de cet exposé, permettez-moi de suggérer certains facteurs principaux qui, à mon sens, ont joué et jouent encore pour tenir les employés de bureau à l'écart du mouvement syndical.

\section{a) Désir de quitter les «classes supérieures 》}

Tout d'abond, signalons le fait que plusieurs collets-blancs sont eux-mêmes les enfants de travailleurs industriels qui, souvent à la faveur de lounds sacrifices, ont su donner à leurs enfants l'instruction scolaire nécessaire pour leur permettre de «sortir des rangs des ouvriers manuels». Bon nombre de ces collets-blancs ont acquis une attitude de supériorité, voire même de mépris et d'hostilité envers les travailleurs industriels et leurs institutions, notamment les syndicats. Ce collet-blanc a acquis l'attitude, fortement encouragée par les employeurs, qu'il pourrait «faire son chemin tout seul», qu'il «n'avait pas besoin d'une union», et que le syndicat, bien, c'était une chose réservée aux «classes inférieures »!

\section{b) Désir de s'identifier d̀ la «direction 》}

En même temps, dans plusieurs cas du moins, le collet-blanc s'est convaincu, encore avec l'encouragement de l'employeur, qu'il faisait partie de la «direction de l'entreprise, même s'il occupait un poste peu important et restait très peu au courant de ce qui se passait dans l'administration de l'entreprise. Encore, certains petits privilèges, comme un congé de temps à autre pour assister aux «funérailles de la grand-mère » au Stade de baseball, la tasse du café à 10 heures du matin, le congé-maladie, les 2 semaines de vacances, et bien d'autres ont encouragé le collet-blanc à croire qu'il jouissait d'un statut tout particulier en comparaison avec la masse des travailleurs.

On trouve que le simple fait de travailler PRES DE LA DIRECTION (physiquement), que l'on soit sur le même étage ou que l'on descende dans le même ascenseur que le président de la Compagnie, a joué aussi un rôle en encourageant l'employé de bureau à se considérer «près de la direction» dans un sens plus large, et à s'efforcer de se 
créer des rapports personnels et intimes avec ses supérieurs, le tout faisant partie de ce rêve de «s'avancer par ses propres efforts ».

Dans le passé, il est vrai, les postes supérieurs étaient à la portée du collet-blanc, ou du moins, à la portée de ses ambitions. Pour l'employé de bureau qui cherchait ou rêvait d'atteindre au haut de Téchelle, un syndicat paraissait comme un obstacle fatal à ses ambitions. De toute façon, il n'en avait point besoin. Aujourd'hui, ces rêves commençent à disparaître pour plusieurs collets-blancs.

Mentionnons, à propos de cette question des aspirations des collets-blancs de monter aux postes supérieurs dans l'entreprise, les données fournies par un relevé fait en 1955 par l'American Institute of Management. Dans 23,000 compagnies questionnées sur leur politique en matière de promotions, $51 \%$ ONT AVOUE QU'ELLES DONNAIENT LA PREFERENCE AUX PARENTS, AUX AMIS ET AUX PROTEGES. Ces firmes avouaient que le choix des fonctionnaires supérieurs se faisait selon des critères N'AYANT AUCUN RAPPORT avec les intérêts réels de l'entreprise!

\section{c) Ignorance et préjugés}

Un autre facteur important jouant contre l'organisation syndicale parmi les collets-blancs est le manque d'information de ceux-ci au sujet du syndicalisme, et les préjugés qui découlent de cette ignorance. Ceci est un grave problème surtout dans les bureaux-chefs et usines où aucun syndicat bona fide n'existe parmi les travailleurs d'usine. Ainsi, on trouve plusieurs collets-blanes qui ont a l'esprit ce portrait ridicule du syndicat comme d'une organisation qui déclare la grève à tous les lundis et mercredis pour satisfaire les ambitions personnelles d'un soidisant «chef ouvrier» mythique. Malheureusement, de telles idées fausses et déformées sur la nature, les buts et la conduite des syndicats sont solidement ancrées chez un grand nombre de collets-blancs et on ne parvient pas à les faire disparaître du soir au lendemain.

LE SYNDICALISME: COMMENT PEUT-IL AIDER LES EMPLOYÉS DE BUREAU?

J'arrive maintenant au dernier et plus important aspect de la question ici discutée.

Etant donné la situation que j’ai décrite, de la disparition graduelle des illusions du collet-blanc sur les possibilités d'atteindre au haut de l'échelle, et surtout de la conscience du fait que ses salaires et conditions de travail traînent en arrière de ceux gagnés par les travailleurs manuels syndiqués, il n'y a rien d'étonnant de voir le collet-blanc accepter de plus en plus pour son propre bénéfice l'idée de l'organisation syndicale. Après tout, malgré ses idées déformés sur les syndicats, le 
collet-blanc est au courant de ce qui se passe autour de lui. Il sait que TOUT LE MONDE est organisé. Sa propre compagnie est membre de l'Association des manufacturiers, un ORGANISME qui agit au nom de ses membres afin d'atteindre certains buts COLLECTIFS. Les ingénieurs professionnels, eux aussi, ont leur propre organisation qui travaille pour améliorer les salaires de ses membres. Même le gérant du personnel fait partie d'un groupement professionnel! Et il sait ce que les travailleurs syndiqués dans l'usine ont pu gagner grâce à leur syndicat. En effet, IL N’Y A QUE LE PAUVRE COLLET-BLANC QUI N'EST PAS ORGANISE DANS CE MONDE HAUTEMENT ORGANISE!

En même temps, plusieurs employés de bureau ont des problèmes au travail, ils ont des griefs. A part et en plus de la question du salaire et des conditions de travail, il existe la question brûlante de la SECURITE D'EMPLOI. J'entends, par ce terme, non seulement lemaintien et ou la perte de son poste, mais aussi la question de promotion, d'augmentations de salaire, de transfert à un emploi plus intéressant, et ainsi de suite. Un homme qui compte déjà 15 années de service comme commis intermédiaire et qui voit un employé avec moins de service lui passer par-dessus le dos est un homme avec un grief, que ce soit justifié ou non. Comment peut-il avoir justice? Par ses propres efforts, en soulevant la question devant ses supérieurs? Oui, il l'a déjà essayé! Mais le surveillant lui en veut, ou encore tient à favoriser le cousin de sa femme, ou il y a autre chose... L'employé ressent l'injustice. Or, ce sont précisément de tels problèmes que vise la procédure de règlement des griefs dans la convention collective, et c'est là que le syndicalisme peut faire beaucoup pour les employés de bureau.

Il va sans dire qu'un des buts primordiaux des syndicats est l'amélioration des salaires et des conditions de travail des employés de bureau. Mais j'insiste sur le fait que, MEME SI LES SALAIRES ET LES AUTRES CONDITIONS MONETAIRES SONT SATISFAISANTS, LE PROBLEME DE LA SECURITE D'EMPLOI RESTERA SANS SOLUTION TANT ET AUSSI LONGTEMPS QUE L'EMPLOYE DE BUREAU ESSAIERA DE FAIRE SON CHEMIN TOUT SEUL. Même une clause relativement anodine (et inadéquate, pour le syndicat) et une procédure de règlement des griefs dans une convention collective fourniront beaucoup plus de protection dans les domaines de la sécurité d'emploi, des promotions, des augmentations de salaires au mérite et ainsi de suite, que si l'on se fie uniquement à la seule discrétion d'un surveillant.

Je peux citer le cas d'un bureau dans une importante usine à Montréal, où une campagne d'organisation syndicale parmi les employés de bureau n'avançait guère jusqu'au moment où des employés: furent congédiés sans égard à leur anciennté. Ce geste produisit une 
forte réaction immédiate parmi ces employés. Et dès lors l'Union fut en mesure de faire entrer les employés dans le syndicat, et de signer peu après une convention collective.

Les syndicats ont fait des progrès considérables en vue de rendre la structure des salaires plus équitable. La «progression automatique » du salaire jusqu'au maximum de l'échelle ou, plus souvent, la progression partielle ainsi que la procédure de règlement des griefs dans le cas des augmentations «de mérite », voilà deux dispositions importantes qui fournissent une protection aux employés contre l'arbitraire et la discrimination!

\section{CoNCLUSION}

On a souvent entendu des employés de bureau parler de la « dignité » de leur fonction, «dignité » qui les empêcherait d'adhérer à un syndicat. Mais, si paradoxal que la chose puisse paraître, l'employé de bureau ne parvient à la pleine dignité de son travail que lorsqu'il fait partie d'un syndicat. Car c'est le syndicat, par l'entremise de la négociation collective, qui permet à l'employé de bureau de garder la tête haute, fort de la connaissance que ses droits sont protégés, que ses mérites seront pris en considération et qu'il peut chercher une solution à ses griefs par une procédure ordonnée de règlement des griefs. prévue dans la convention collective.

(Causerie prononcée devant le Montreal Board of Trade, le 20 mars 1958.)

The State of Labor-Management Relations, 1958-1959

\section{Arthur J. Goldberg}

After explaining Sidney Hillman's contribution to labor philosophy and to improved labor-management relations, the author draws a vivid picture of the present state of labor-management relations. Hillman believed in the mutuality of interests between both sides, but there was no question of surrendering to Management. We are a long way from a universal achievement of the type of labor-management relations symbolysed by the Sidney Hillman's tradition. Union organization meets with resistance; all parties toughen their attitudes and restrict their meetings to those around the bargaining table. To bridge the gap and introduce more talks on basic issues between sides, a Labor-Management Assembly is proposed.

The name of Sidney Hillman creates what the folk on Madison Avenue are fond of describing as a clear and definite «image». $\mathrm{He}$ 